Annuaire suisse de politique de développement

22-2 | 2003

Société de l'information et coopération internationale

\title{
Gouvernance de l'Internet : une centralisation du pouvoir inquiétante
}

\section{Stéphane Koch}

\section{(2) OpenEdition}

1 Journals

Édition électronique

URL : http://journals.openedition.org/aspd/585

DOI : 10.4000/aspd.585

ISSN : 1663-9669

Éditeur

Institut de hautes études internationales et du développement

\section{Édition imprimée}

Date de publication : 1 novembre 2003

Pagination : 189-194

ISSN : 1660-5934

\section{Référence électronique}

Stéphane Koch, « Gouvernance de l'Internet : une centralisation du pouvoir inquiétante », Annuaire suisse de politique de développement [En ligne], 22-2 | 2003, mis en ligne le 23 mars 2010, consulté le 08 septembre 2020. URL : http://journals.openedition.org/aspd/585 ; DOI : https://doi.org/10.4000/ aspd.585 


\title{
Gouvernance de l'Internet: une centralisation du pouvoir inquiétante
}

\author{
Stéphane Koch*
}

$\mathrm{N}$ é d'une initiative militaire pour répondre à des besoins de communication d'ordre stratégique à l'échelon national, le Net a très vite dépassé l'idée première de ses concepteurs. Originellement, le réseau devait d'abord répondre à un cahier des charges purement technique: résister à une attaque nucléaire et conserver un système de communication opérationnel. Par la suite, la communauté scientifique s'est emparée du modèle pour ses propres besoins.

Au-delà d'une gestion «technique» du protocole IP et de l'architecture du Net - dont le développement et la standardisation ont été confiés à l'IETF (Internet Engineering Task Force) en 1986, avant que le processus soit légalisé par la création de l'ISOC (Internet Society) en 1992 -, le besoin d'une régulation plus «politique» ne s'était pas fait sentir jusqu'alors. Personne n'avait anticipé l'engouement qu'allait connaître le Net. Les communautés scientifiques et académiques de même que les autres entités participatives du réseau n'avaient pas ressenti la nécessité de mettre en place une régulation globale du fonctionnement de la «toile». Elles avaient en effet déjà des règles et des modes de fonctionnement opérationnels, lesquels correspondaient aux besoins d'un modèle non commercial et partagé, dont les diverses parties prenantes se satisfaisaient.

C'est le développement du world wide web entre 1989 et 1993 - grâce à la naissance de l'hypertexte et du navigateur Mosaic - qui a permis à l'Internet de prendre son essor planétaire. Dès ce moment, la machine s'est emballée et le réseau mondial s'est étendu de manière exponentielle, balayant les modèles de distribution de l'information classiquement établis. L'éclatement des frontières géographiques a favorisé l'émergence de communautés numériques, ciment de la «société de l'information» que l'on connaît aujourd'hui.

C'est dans ce contexte spécifique qu'est apparu le premier organisme de gestion des noms de domaine: l'IANA (Internet Assigned Numbers Authority). Créée par John Postel, elle avait pour but de servir d'interface entre les adresses IP et les noms de domaine, de gérer les extensions de domaine génériques telles que, entre autres, les .org, .com et .net ainsi que les différentes classes d'adresses IP. De fait, l'IANA était une réponse à un besoin plus technique que politico-communautaire. Il a donc fallu développer un modèle correspondant à l'essor fulgurant du web. C'est ainsi qu'en 1998 un autre organisme, l'ICANN (Internet Corporation for Assigned Names and Numbers), va naître d'un consensus entre le 
gouvernement américain et quelques associations représentatives de l'Internet (ISOC, IAB [Internet Architecture Board], IETF...) pour suppléer aux déficiences naturelles de l'IANA.

L'ICANN, société de droit californien à but non lucratif et indépendante, avait pour tâche de répondre à un certain nombre d'attentes générées par l'explosion du développement de l'Internet. Le but affirmé était d'offrir plus de transparence et de démocratie dans les décisions liées au développement du Net. En plus de la coordination technique du système des noms de domaine, de l'allocation des adresses IP ainsi que de la gestion des serveurs racines, l'ICANN était censée aplanir les problématiques de monopole et mettre en place une gestion plus ouverte des ressources liées aux noms de domaine.

Dès sa création, l'organisme a connu un certain nombre de critiques. D'abord, il n'abordait pas certaines dimensions comme les libertés civiles et la protection de la sphère privée. Ensuite, il n'offrait pas d'accès public à ses travaux. Enfin, son indépendance affirmée était mise en doute: le fait que l'ICANN soit liée au Département du commerce américain va en effet à l'encontre des attentes de la communauté internationale. D'autant plus que plusieurs pays - majoritairement du Sud - y voient une ingérence des Etats-Unis dans leurs affaires. Ces pays n'acceptent pas de devoir rendre des comptes au Département du commerce pour l'obtention de leur propre ccTLD (code des noms de domaine par pays). Le droit de veto octroyé au Département du commerce lors de la création de l'ICANN lui confère un pouvoir de pression énorme qui lui permet de se positionner comme l'organe de contrôle ou de «conseil» de la structure de gestion des noms de domaine. On imagine mal que l'ICANN fasse cavalier seul ou prenne des décisions importantes qui n'iraient pas dans le sens des attentes de Washington. Lors d'un accord conclu récemment avec l'ICANN, le Département du commerce a vu ses pouvoirs accrus; il a obtenu la possibilité de contrôler tous les trimestres le travail de réforme de l'ICANN ainsi que de participer à des travaux ayant pour objectif d'offrir plus de pouvoir aux gouvernements.

Voulant afficher une certaine transparence, l'ICANN a, lors de sa constitution, organisé un vote par Internet dans le but de faire élire par la communauté online les représentants continentaux (Afrique, Asie/Pacifique, Europe, Amérique latine et Caraïbes, Amérique du Nord) appelés à siéger dans son comité directeur. A l'origine, ils étaient même censés occuper jusqu'à la moitié des sièges disponibles. Même si le modèle de vote a connu quelques problèmes techniques, engendrant un certain chaos, l'idée n'en restait pas moins valable et démocratique. Cependant, l'ICANN a décidé récemment de redéfinir son modèle et supprimé du processus décisionnel les représentants élus par la communauté Internet. Le nombre de personnes siégeant au comité directeur a été réduit de 19 à $15 ; 8$ de celles-ci sont désignées par un comité de nomination composé des représentants des gouvernements, des utilisateurs commerciaux et des organisations à but non lucratif, le solde étant choisi par les délégués des structures de gestion des noms de domaine «génériques» (TLD) et «nationaux» (ccTLD) et des bureaux d'enregistrement. Le comité At Large a quant à lui, après avoir voté en faveur de ce «seppuku démocratique», été enrichi de quelques participants pour se composer au final de 10 membres «conti- 
nentaux» et de 5 autres membres nommés par l'ICANN et le comité de nomination. En résumé, plus de membres, avec moins de pouvoir décisionnel - le comité At Large n'occupant plus qu'une fonction de conseil.

Dès ses débuts, l'ICANN a été victime d'une prise de pouvoir par certains groupes d'intérêts économiques comme Network Solution (Verisign), en charge de gérer les extensions génériques - dont le très lucratif .com. Forts de leur pouvoir financier, ces groupes ont exercé une influence importante dans les négociations et les décisions visant à mettre en place la nouvelle structure de gestion des noms de domaine. Bien que se voulant indépendante et à but non lucratif, l'ICANN n'a pas été en mesure de faire barrage à l'application de modèles d'économie de marché, qui ont privilégié les spéculateurs et autres cybersquatters au détriment du reste de la communauté. On aurait pu en effet s'attendre à voir apparaître un certain nombre de règles visant à un développement de l'Internet sur le long terme et favorisant une répartition homogène des ressources. Or, il faut se rendre à l'évidence que la réalité est tout autre.

Les pays du Sud ont été les premiers à payer le prix d'une politique orientée vers le marché. Souffrant déjà d'un retard technologique lié au coût d'acquisition et de mise en place des infrastructures de transport de l'information, certains d'entre eux ont en plus été spoliés des possibilités de l'exploitation durable de leur nom de domaine national. L'exemple de Tuvalu, .tv, est représentatif de cette problématique. Cet archipel perdu au milieu du Pacifique aurait pu ou dû bénéficier d'un accompagnement, afin de mettre en place un modèle de développement économique durable lié à la gestion commerciale de son nom de domaine, celui-ci étant extrêmement attractif pour le secteur de la télévision. Or, c'est une société américaine qui s'est emparée de ce nom de domaine en échange de quelques dizaines de millions de dollars. On ne peut donc que condamner le fait que l'ICANN n'ait pas réfléchi à une protection contre ce genre d'agissements.

Le multilinguisme est une autre illustration du manque de prise en compte des intérêts des pays tiers. L'ICANN a décidé de créer une interface permettant de gérer les «caractères spéciaux» tels que, entre autres, les accents dans les langues occidentales ou les caractères asiatiques. Dès que le modèle a plus ou moins fonctionné au niveau technique, on s'est empressé de vendre ce «nouveau produit» - en fait, jusqu'au milieu de l'année 2002, seules des options d'achat ont été vendues par Verisign. Comme aucune mesure n'avait été prévue pour prévenir les conflits liés aux noms de domaine et que, dans les langues asiatiques, des noms s'écrivant de la même manière peuvent avoir plusieurs significations, de nombreux litiges ont vu le jour. Ceux-ci ont été réglés par le centre d'arbitrage de l'Organisation mondiale de la propriété intellectuelle $(\mathrm{OMPI})$, après règlement des frais.

Cette manière de procéder représente une discrimination supplémentaire pour les pays du Sud, qui doivent déjà affronter un fossé technologique que seuls couvrent, sans le combler, les discours politiques. Le manque de volonté des gouvernements des pays dits développés, le laxisme de l'industrie et la quête d'hégémonie de certains pays risquent de créer à court terme une situation iden- 
tique à celle que connaissent déjà les pays du Sud au niveau de l'exploitation de leurs ressources naturelles. Dans les faits, on assiste à l'acceptation tacite d'une situation dont les conséquences seront, à terme, la vassalisation d'un certain nombre d'ethnies, de populations ou de cultures. Cela alors même que la société dite de l'information était annoncée comme une plate-forme d'échanges multiculturels.

Annoncé comme un modèle décentralisé de distribution de l'information, l'Internet souffre donc d'une centralisation du pouvoir inquiétante. Les noms de domaine et les serveurs qui les gèrent sont les seules ressources qui soient entièrement centralisées. Sur les 14 serveurs racines répartis dans le monde, seuls 4 ne sont pas situés aux Etats-Unis - ils se trouvent au Japon, en Angleterre, en Espagne et en Suède. Les deux tiers des ordinateurs connectés à Internet sont localisés aux Etats-Unis, et les principales liaisons à haut débit entre les EtatsUnis et l'Europe occidentale passent par Londres. Paradoxalement, les pays du Sud et les pays asiatiques paient un tribut élevé pour accéder au réseau «gratuit». Les échanges d'informations entre les serveurs-racines et les détenteurs de noms de domaine représentent un coût de l'ordre de plusieurs dizaines de millions d'euros par année. On peut noter que 80 à $90 \%$ de ces paquets d'informations qui transitent sur Internet passent par les Etats-Unis, et que presque $90 \%$ des ordinateurs utilisent Microsoft.

De récents événements ont démontré la nécessité d'une répartition stratégique des ressources nécessaires au fonctionnement de l'Internet: entre le 21 et le 22 octobre 2002, les serveurs racines ont subi des attaques simultanées par deny of service - attaques par de multiples requêtes qui engorgent les serveurs. Bien que le fonctionnement même de l'Internet n'ait pas été mis en danger, on a pu constater une diminution de la capacité à traiter le volume des échanges habituels, à commencer par les connexions les plus éloignées des autoroutes de l'information. Les auteurs de ces attaques n'ont jusqu'ici pas été identifiés; cependant, de nombreux spécialistes pensent que l'origine d'une telle action est liée à la forme actuelle de la politique étrangère américaine. A cet égard, on peut citer l'exemple du virus Slammer, diffusé sur le Net le 25 janvier 2003 : il s'attaquait uniquement à des machines hébergeant des produits (bases de données) de la société Microsoft.

Il faut se rendre compte que le traitement des données personnelles, leur utilisation à des fins commerciales, gouvernementales ou militaires, ne font à l'heure actuelle l'objet d'aucune protection efficace. Cette centralisation de l'Internet a permis aux Etats-Unis de s'approprier le traitement des informations qui transitent à travers le monde. Sous le couvert d'une lutte contre le terrorisme, de plus en plus de données sont collectées sans que leurs titulaires en soient vraiment conscients ou même informés. DARPA (Defense Advanced Research Projects Agency), le centre de recherche de l'armée américaine, illustre bien cette tendance par la création de l'IAO (Information Awareness Office), dont le but est de gérer et d'interpréter les informations collectées.

La mondialisation des échanges crée une dépendance croissante des sociétés dites «développées» envers les systèmes d'information. Le principe de la plate- 
forme d'échanges que représente Internet bascule peu à peu vers une entité multidimensionnelle de gestion de flux d'informations hétérogènes. Les dimensions multiculturelles et communautaires s'estompent pour laisser la place à d'autres aspects et notions. La gestion des interdépendances technologiques génère une nouvelle dynamique des influences politiques; le contrôle de l'infosphère représente en effet un avantage stratégique indéniable dans l'état actuel de guerre économique et de conquête des marchés. Il est dès lors difficile d'envisager une volonté sincère de "partager et distribuer» les capacités du réseau mondial. Aujourd'hui, seuls les rapports de force régissent notre société; on est loin des visions sur le renouveau de l'expression démocratique et des politiques en cours promis par l'Internet. Au contraire, on assiste à une volonté de marginalisation de l'expression des mouvements de lutte contre la mondialisation des économies. La société de consommation privilégie la pensée unique au détriment de la société elle-même, malgré l'émergence de nouveaux mouvements citoyens grâce à l'abolition des frontières classiques par les déferlantes numériques de la société en réseau. L'Internet est de facto le miroir de la société que nous construisons. Son développement nécessite les mêmes valeurs morales et une responsabilisation sociale.

IPv6 (Internet Protocol version 6) pourrait représenter une alternative à la répartition actuelle des ressources. Cette nouvelle version du protocole Internet (TCP/IP) devrait permettre, techniquement, de relancer le débat politique visant à une distribution plus homogène des serveurs racines sur les différents continents. Cela dit, le gouvernement américain a prévu que l'ensemble du Département de la défense serait opérationnel avec IPv6 d'ici à 2008. On peut donc estimer que si l'Europe et l'Asie ne favorisent pas prioritairement la mise en œuvre de ce nouveau protocole, il leur sera difficile de constituer un contrepoids suffisant. De plus, ce nouveau protocole permettra de hiérarchiser les données et de les traiter de manière choisie; on pourra traiter des flux d'informations commerciaux (vidéos et technologies de type rich media) plus rapidement, au détriment des informations circulant encore sous le protocole actuel. Les pays en développement - qui peinent déjà à mettre en place une «infrastructure» - vont une fois de plus souffrir de l'avancée technologique des pays industrialisés. La gestion du protocole IPv6 demande en effet un matériel de pointe, que seules les entités commerciales sont à même de s'offrir. On ne fera donc qu'accroître le déséquilibre déjà existant, puisque l'on équipe aujourd'hui les pays défavorisés avec un matériel que l'on sait inadapté pour le développement futur du Net.

De plus, à moyen terme, cette nouvelle version du protocole représente un danger stratégique pour la position de l'UIT (Union internationale des télécommunications). IPv6 permettra de gérer spécifiquement la téléphonie par Internet. Celle-ci était jusqu'alors gérée au travers des infrastructures de télécommunications conventionnelles, dont la définition des règles - techniques et politiques - régulant les échanges était l'apanage de l'UIT. Le trafic des informations transportées par le biais d'IPv6 repose sur la gestion de la distribution des adresses IP et de leur routage, et ceux-ci sont déjà gérés par l'ICANN. L'avenir étant au «tout IP», les réseaux de téléphonie finiront irrémédiablement par basculer dans le voice over IP. On peut donc avoir des craintes légitimes concernant la gestion future des infrastructures de la téléphonie mondiale. 
A la fin 2003, le Sommet mondial sur la société de l'information se tiendra à Genève. Cet événement se voulait fédérateur des différentes composantes de la société civile, du secteur privé et des gouvernements, un catalyseur de leur mise en réseau. Il semble qu'il se destine plus à être le symbole de la problématique abordée dans ce texte. La Tunisie, pays initiateur et futur pays hôte (en 2005), n'a selon toute vraisemblance pas encore compris la relation qui existait entre "société de l'information», liberté d'expression et démocratie; pendant la préparation du sommet de Genève, elle a en effet emprisonné plus de 20 personnes dont le «crime» a été de visiter des sites web interdits par leur gouvernement. Les droits de l'homme, la liberté d'expression, l'accès à l'information, le droit à l'information sont aussi des sujets qui irritent bon nombre d'Etats. Malgré la volonté et le travail considérable des diverses composantes de la société civile pour apporter une contribution positive et constructive à cet édifice qu'est la société de l'information, elles seront écartées des processus décisionnels; ceuxci vont rester l'apanage de gouvernements qui, dans leur majorité, voient d'un mauvais œil cette «ingérence citoyenne».

Le sommet sera une plate-forme stratégique pour son principal organisateur, l'UIT. Celle-ci a perdu pas mal de terrain ces dernières années sur le plan de la société numérique, notamment à cause de ses lourdeurs administratives et d'un certain manque de clairvoyance. Ces derniers mois, l'organisation a multiplié les conférences, séminaires et autres débats sur la gestion des noms de domaine, démontrant une volonté de changer le consensus actuel que représente l'ICANN. On dénote une volonté de la part de l'UIT de prendre le contrôle des noms de domaine nationaux. Le Département du commerce américain, par sa présence dans les événements organisés par l'UIT, illustre bien l'importance de cette bataille silencieuse et son but inavoué: garder le contrôle des noms de domaine.

Tant que nous laisserons le pouvoir politique agir à sa guise - paradoxalement contre l'intérêt de ses électeurs -, aucune solution ne sera envisageable. Seule une réelle prise de conscience par les citoyens des impacts réels de la société de l'information dans leur vie de tous les jours pourra créer le ciment nécessaire pour construire les bases solides d'une société numérique dont nous ne serons pas de simples utilisateurs, mais les composantes. 